\title{
Search for Charged Lepton Flavour Violation at CMS
}

\author{
Swagata Mukherjee ${ }^{1, a}$ \\ On behalf of the CMS Collaboration \\ ${ }^{1}$ Physics Institute IIIA, RWTH Aachen University, Germany
}

\begin{abstract}
Lepton flavour is a conserved quantity in the standard model of particle physics, but it does not follow from an underlying gauge symmetry. After the discovery of neutrino oscillation, it has been established that lepton flavour is not conserved in the neutral sector. Thus the lepton sector is an excellent place to look for New Physics, and in this perspective the Charged Lepton Flavour Violation is interesting. Various extensions of the standard model predict lepton flavour violating decays that can be observed at LHC. This report summarises several searches for lepton flavour violation with data collected by the CMS detector.
\end{abstract}

\section{Introduction}

Lepton flavour conservation in the standard model (SM) of particle physics does not follow from any underlying gauge symmetry. From neutrino oscillation, its violation in the neutral sector is already known. Neutrino oscillation allows, in principle, charged lepton flavour violation (LFV) but the large mass difference between neutrinos and heavy gauge bosons suppresses such interactions to orders of magnitude, which is immeasurable by any existing experimental setup. However, extensions of the SM can lead to significant enhancements of processes involving charged LFV. Charged LVF processes can be directly related to neutrino masses and mixing and therefore are very promising to find evidence for physics beyond the SM. At CMS [1], several searches for charged LFV have been performed in different channels and final state topologies. Search for heavy majorana neutrinos and R-parity violating (RPV) supersymmetry (SUSY) are particularly important. Another interesting scenario is where LFV Higgs boson decays become amplified in models assuming the validity of the SM only up to a finite scale. Other possibilities are models involving more than one Higgs doublet or models predicting leptoquarks.

During LHC Run-I, a multitude of searches for the charged LFV have been performed by the CMS collaboration at proton-proton centre-of-mass energies of 7 and $8 \mathrm{TeV}$. The increase of the energy in Run-II to $\sqrt{s}=13 \mathrm{TeV}$ has lead to a substantial increase in sensitivity. As a consequence, the mass reach for some of the searches was substantially extended after the analysis of data taken in 2015 . However, this was not true for all models since the integrated luminosity recorded in 2015 was only about 1/10th of the one of Run-I. In 2016, the LHC did a fantastic job, exceeding expectations, reaching an instantaneous luminosity of $1.5 \times 10^{34} \mathrm{~cm}^{-2} \mathrm{sec}^{-1}$. The CMS experiment collected data with more than $92 \%$ efficiency and approximately $36 \mathrm{fb}^{-1}$ of integrated luminosity were collected for

a e-mail: mukherjee@physik.rwth-aachen.de 
physics. The high instantaneous luminosity comes at the price of pileup, the number of overlapping proton-proton interactions in one bunch crossing. In 2016 an average of 24 pileup interactions was observed. However, CMS have developed sophisticated tools to mitigate the effect of pileup at the trigger and object reconstruction level.

In this report, some of the important searches related to charged LFV with the CMS experiment is described in brief.

\section{Lepton flavour violating decays of Higgs boson}

In SM, the LFV decays are forbidden if the theory has to be renormalizable. If this requirement is relaxed, so the theory is valid only to a finite mass scale, then LFV couplings may be introduced. LFV decays can occur naturally in models with more than one Higgs doublet without abandoning renormalizability. They also arise in supersymmetric models, composite Higgs boson models, models with flavour symmetries, Randall-Sundrum(RS) models and many others. The presence of LFV couplings would allow $\mu \rightarrow e, \tau \rightarrow \mu$ and $\tau \rightarrow e$ transitions to proceed via a virtual Higgs boson.

\section{$2.1 H \rightarrow \mu \tau$}

The search is based on a dataset of $35.9 \mathrm{fb}^{-1}$ of proton-proton collisions collected with the CMS detector in 2016, at a center-of-mass energy of $13 \mathrm{TeV}$ [2]. The decay channel, $H \rightarrow \mu \tau_{\mu}$, is not
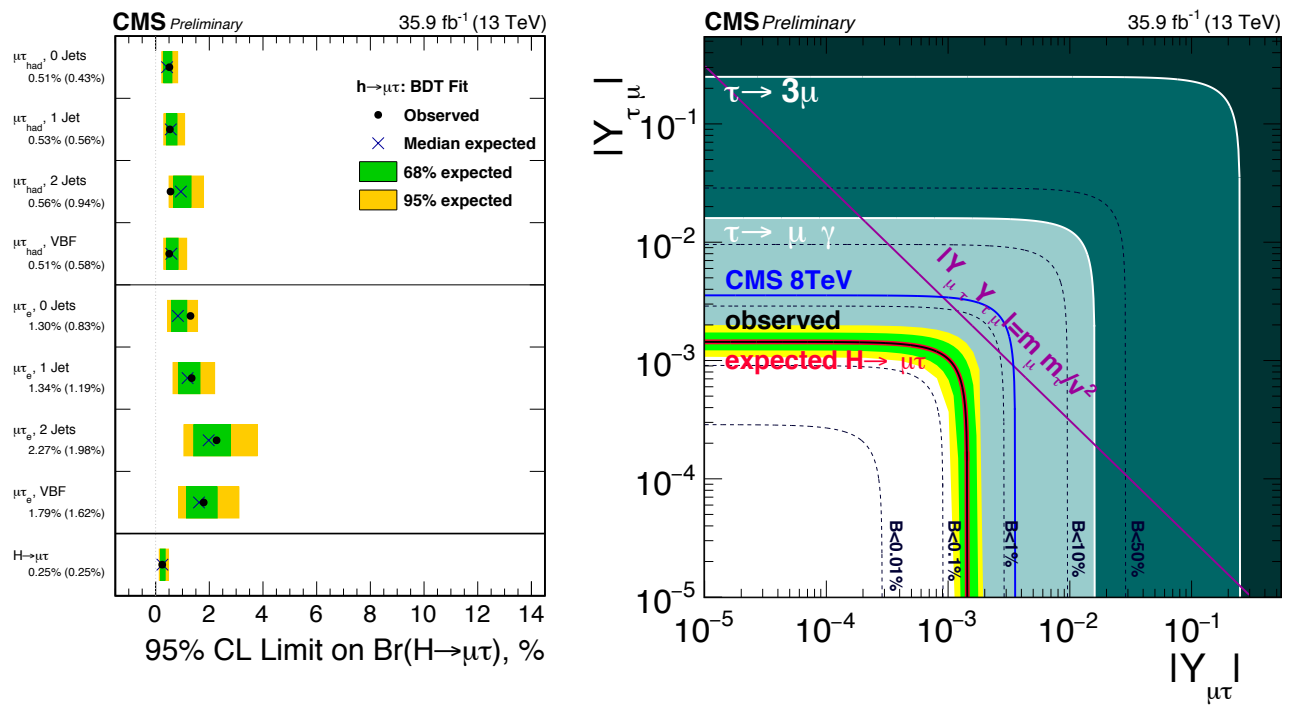

Figure 1. Left: Observed and expected $95 \%$ CL upper limits on the $B(H \rightarrow \mu \tau)$ for each individual category and combined [2]. Right: Constraints on the flavour violating Yukawa couplings, $\left|Y_{\mu \tau}\right|$ and $\left|Y_{\tau \mu}\right|$ [2]. The expected (red solid line) and observed (black solid line) limits are derived from the limit on $B(H \rightarrow \mu \tau)$. The flavour diagonal Yukawa couplings are approximated by their SM values. The green (yellow) band indicates the range that is expected to contain $68 \%$ (95\%) of all observed limit excursions from the expected limit. The shaded regions are derived constraints from null searches for $\tau \rightarrow 3 \mu$ (dark green) and $\tau \rightarrow \mu \gamma$ (lighter green).The purple diagonal line is the theoretical naturalness limit.

considered due to the large background contribution from $Z \rightarrow \mu \mu$ decays. The indirect constraint, 
$B(H \rightarrow \mu \tau)<O(10 \%)$ is not very stringent, hence this direct search is very important. In this search, a loose selection is first applied, and then the events are divided into categories according to the number of jets in the event. The 0 -jet category enhances the gluon fusion contribution and the 1-jet category enhances gluon fusion production with initial state radiation. The 2-jet gluon fusion category has a further requirement that the invariant mass of the two jets $M_{\mathrm{jj}}<550 \mathrm{GeV}$ while the 2-jet VBF category with the requirement $M_{\mathrm{jj}}>550 \mathrm{GeV}$ enhances the VBF contribution. After the loose selection a set of eight kinematic variables is combined into a boosted decision tree (BDT). The signal used for training the BDT is a mixture of simulated gluon fusion and VBF events, weighted according to their respective production cross sections. In $\mu \tau_{h}$ channel, the background used for training is a collision data sample of misidentified lepton events. This is the dominant background in this channel. In $\mu \tau_{e}$ channel, the background used for training is a mixed sample of $t \bar{t}$ and Drell-Yan events weighted by their cross-sections. A binned likelihood is used to fit the distributions of the BDT output for the signal and the background contributions. No significant excess over the standard model background expectation is observed. The observed (expected) upper limits on the branching fraction of the Higgs boson are found to be $B(H \rightarrow \mu \tau)<0.25(0.25) \%$ The new results exclude the branching fraction that corresponded to the best fit for the $2.4 \sigma$ excess observed in the $8 \mathrm{TeV} H \rightarrow \mu \tau$ channel at 95\% CL. The observed and median expected 95\% CL upper limits are given for each category in Figure 1-left. The constraints on branching fraction can be interpreted in terms of LFV Yukawa couplings, as shown in Figure 1-right.
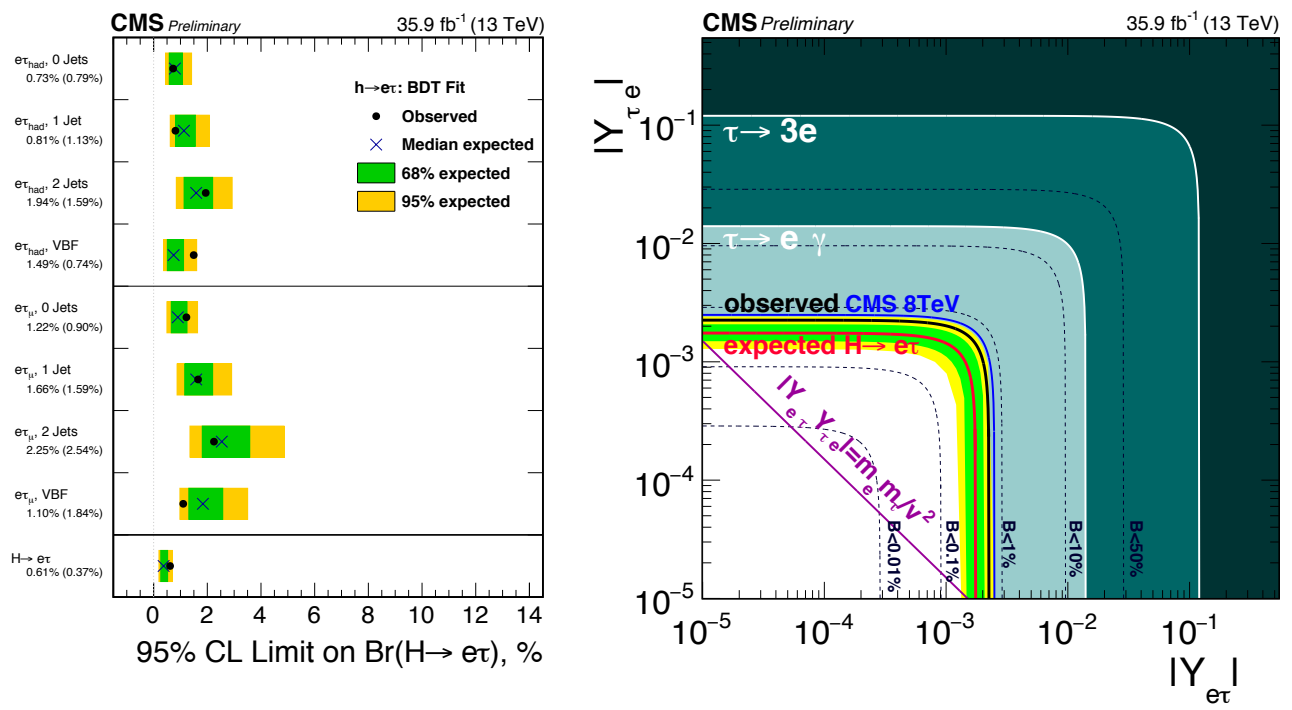

Figure 2. Left: Observed and expected $95 \% \mathrm{CL}$ upper limits on the $B(H \rightarrow e \tau)$ for each individual category and combined [2]. Right: Constraints on the flavour violating Yukawa couplings, $\left|Y_{e \tau}\right|$ and $\left|Y_{\tau e}\right|$ [2]. The expected (red solid line) and observed (black solid line) limits are derived from the limit on $B(H \rightarrow \mu \tau)$. The flavour diagonal Yukawa couplings are approximated by their SM values. The green (yellow) band indicates the range that is expected to contain $68 \%$ (95\%) of all observed limit excursions from the expected limit. The shaded regions are derived constraints from null searches for $\tau \rightarrow 3 e$ (dark green) and $\tau \rightarrow e \gamma$ (lighter green).The purple diagonal line is the theoretical naturalness limit. 


\section{$2.2 H \rightarrow e \tau$}

The search is based on $35.9 \mathrm{fb}^{-1}$ data collected with CMS in 2016, at a center-of-mass energy of 13 $\mathrm{TeV}$ [2]. The decay channel, $H \rightarrow e \tau_{e}$, is not considered due to the large background contribution from $\mathrm{Z}$ boson decays. The indirect constraint, $B(H \rightarrow e \tau)<O(10 \%)$ is not very stringent. The $\mathrm{BDT}$ analysis strategy is very similar to the $\mu \tau$ analysis. The same categorisation is performed here as well. In $e \tau_{h}$ channel, the similar BDT training samples as $\mu \tau_{h}$ analysis are used. In $e \tau_{\mu}$ channel, the background used for BDT training is a sample of simulated $t \bar{t}$ events. No significant excess over the standard model background expectation is observed. The observed (expected) upper limits on the branching fraction of the Higgs boson are found to be $B(H \rightarrow e \tau)<0.61(0.37) \%$. The observed and median expected 95\% CL upper limits are given for each category in Figure 2-left. The constraints on branching fraction can be interpreted in terms of LFV Yukawa couplings, as shown in Figure 2-right.

$2.3 H \rightarrow e \mu$

A direct search for lepton flavour violating decays of the Higgs boson in the $H \rightarrow e \mu$ channel is performed, using $19.7 \mathrm{fb}^{-1}$ data collected by CMS at $\sqrt{s}=8 \mathrm{TeV}$ in 2012 [3]. The null result
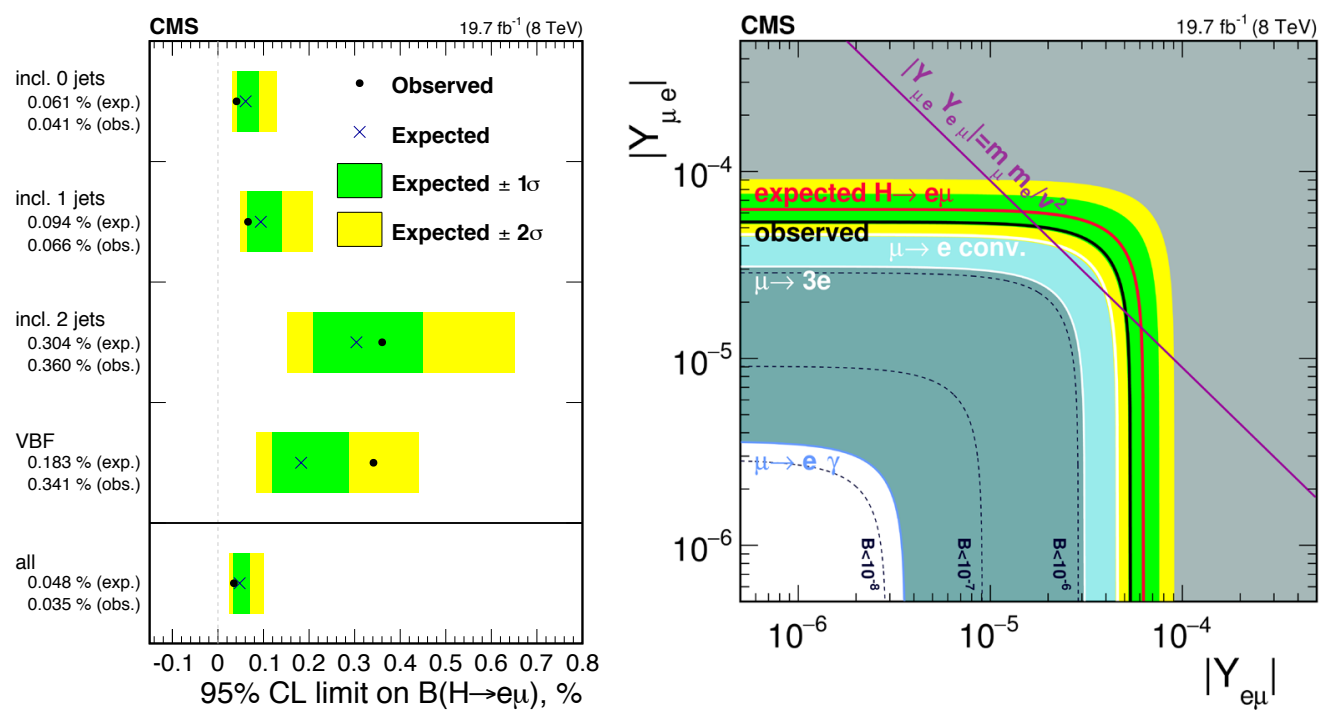

Figure 3. Left: $95 \% \mathrm{CL}$ upper limits by category for $H \rightarrow e \mu$ decay for categories combined by number of jets, the VBF categories combined and all categories combined [3]. Right: Constraints on the flavour violating Yukawa couplings $\left|Y_{e \mu}\right|$ and $\left|Y_{\mu e}\right|$ [3]. The expected (red solid line) and observed (black solid line) limits are derived from the limits on $B(H \rightarrow e \mu)$ from the present analysis. The flavour diagonal Yukawa couplings are approximated by their SM values. The green (yellow) band indicates the range that is expected to contain $68 \%$

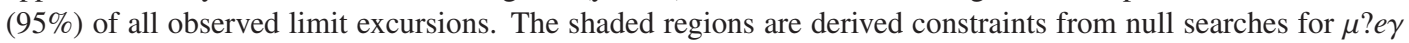
(dark green), $\mu \rightarrow 3 e$ (light blue) and $\mu \rightarrow e$ conversions (grey). The purple diagonal line is the theoretical naturalness limit.

for $\mu \rightarrow$ e $\gamma$ strongly constrains this channel, $B(H \rightarrow e \mu)<O\left(10^{-8}\right)$. However, this limit can be degraded by the cancellation of lepton flavour violating effects from other new physics. The direct search for $H \rightarrow e \mu$ decays presented here is therefore complementary to indirect limits obtained from 
searches for rare decays at lower energies. To enhance the signal sensitivity, events are categorised into nine different categories according to the region of detection of the leptons and the number of jets, and further two categories enriched in VBF production. The dominant background contributions in this search are from Drell-Yan production of $\tau$ lepton pairs and electroweak diboson production. No LFV signal observed in this analysis. An exclusion limit on the branching fraction $B(H \rightarrow e \mu)$ with $M_{H}=125 \mathrm{GeV}$ is derived using the CLs asymptotic model. It is shown in Figure 3-left. The expected upper limit is $B(H \rightarrow e \mu)<0.048 \%$ and the observed upper limit is $B(H \rightarrow e \mu)<0.035 \%$ at $95 \%$ confidence level. These limits are used to constrain the corresponding flavour violating Yukawa couplings, as shown in Figure 3-right.

\section{Search for stop pair production}

Natural SUSY models feature pair production of stops that decay to a number of final states. To fully test supersymmetric naturalness, searches for all possible decay chains should be carried out. These can be broadly categorized as R-parity conserving (RPC) or violating (RPV) scenarios, where R-parity is defined by $R=(-1)^{3 B+L+2 s}$, where $B$ and $L$ are the baryon and lepton numbers, and $s$ is the spin of the particle. All SM particle fields have $R=+1$ while all superpartner fields have $R=-1$. When R-parity is conserved, superpartners are produced in pairs, the lightest superpartner (LSP) is stable and a dark-matter candidate, and proton stability is ensured. Supersymmetric models with RPV interactions violate either $B$ or $L$ but can avoid proton decay limits. The RPV interactions allow for single production of SUSY particles (sparticles) and for sparticle decay into SM only particles. To demonstrate how natural SUSY might manifest itself with RPV couplings, a stop RPV model is examined. In this model, the stop decays to a top quark and intermediate on or off shell bino. The
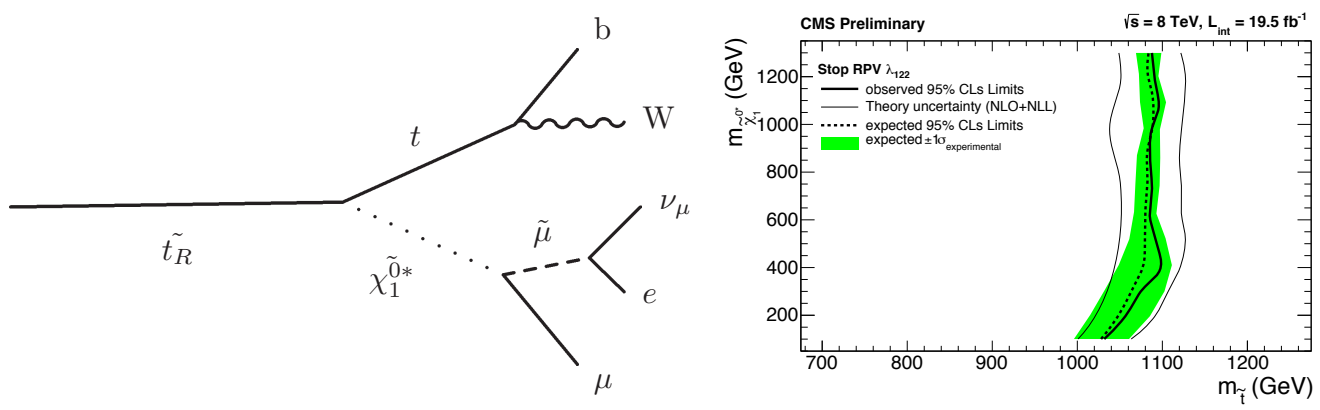

Figure 4. Left: Feynman diagram for RPV coupling $\lambda_{122}$. Right: The $95 \%$ confidence level limits in the stop and bino mass plane for models with RPV coupling $\lambda_{122}$ [4]. The region to the left of the curve is excluded.

intermediate bino then decays to two leptons and a neutrino through the leptonic R-parity violating interaction, as shown in Figure 4-left for RPV coupling $\lambda_{122}$ and Figure 5-left for RPV coupling $\lambda_{233}$. The LFV occurs in the decay of smuon into electron and muon-neutrino as shown in Figure 4-left, and in the decay of stau to muon and tau-neutrino as presented in Figure 5-left. The search looks for events with three or more isolated leptons and bottom-quark jets produced in pp collisions at $\sqrt{s}=8$ $\mathrm{TeV}$ [4]. The analysis is based on a data sample corresponding to an integrated luminosity of 19.5 $\mathrm{fb}^{-1}$ collected by the CMS experiment in 2012. Eight mutually exclusive signal regions (SRs) are defined, depending on the total number of leptons and the number of $\tau_{h}$ candidates in the event. No excess above the standard model expectations is observed in any of the SRs. The limits are mostly 

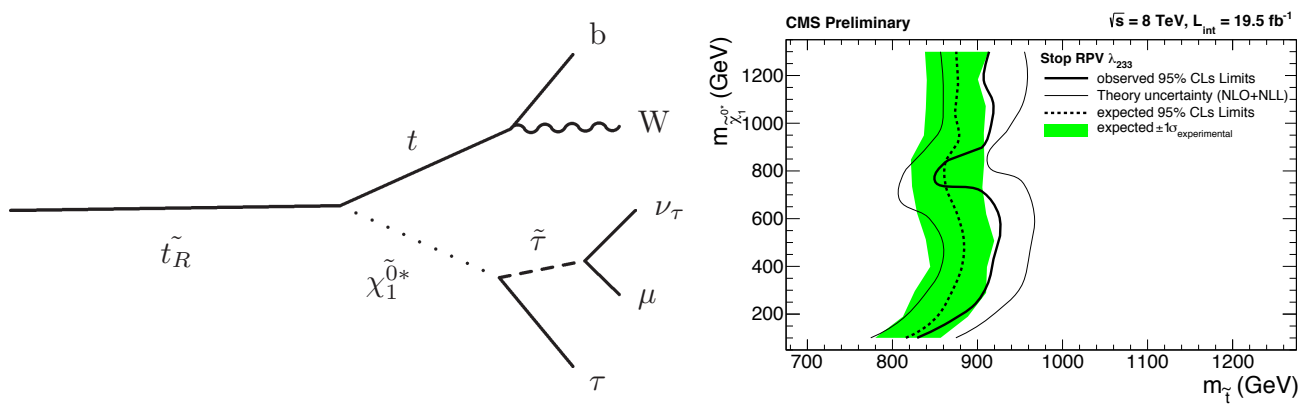

Figure 5. Left: Feynman diagram for RPV coupling $\lambda_{233}$. Right: The $95 \%$ confidence level limits in the stop and bino mass plane for models with RPV coupling $\lambda_{233}$ [4]. The region to the left of the curve is excluded.

independent of the bino mass and are shown in Figure 4-right for $\lambda_{122}$ and in Figure 5-right for $\lambda_{233}$. With the observed result, where the bino mass is $200 \mathrm{GeV}$, models with the stop mass below 1020 $\mathrm{GeV}$ is excluded when $\lambda_{122}$ is non-zero, and below $820 \mathrm{GeV}$ is excluded when $\lambda_{233}$ is non-zero.

\section{High mass $e \mu$ search}

A search for heavy resonances decaying into $e \mu$ final states has been performed using an integrated luminosity of $2.7 \mathrm{fb}^{-1}$ of $13 \mathrm{TeV}$ proton-proton collision data recorded with the CMS detector in 2015 [5]. The search is designed in a model-independent way by only requiring one prompt muon
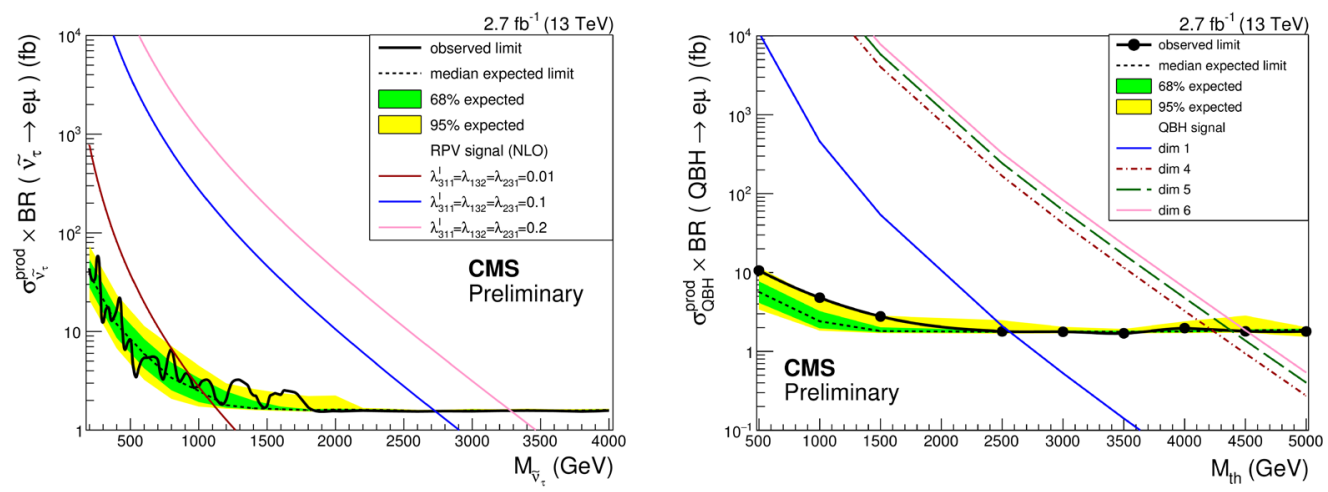

Figure 6. Left: $95 \%$ CL upper limit on the signal cross section times branching fraction for the RPV $\tau$ sneutrino signal as a function of the mass of the resonance [5]. Right: 95\% CL upper limit on the signal cross section times branching fraction for the QBH signal as a function of the threshold mass [5].

and one prompt electron in the event selection. The main sources of background are $t \bar{t}$ and WW processes. No evidence for physics beyond the SM is observed in the invariant mass spectrum of selected $e \mu$ pairs. To interpret the results, two different signal models are used. One is the RPV SUSY, where the $\tau$ sneutrino signal gives rise to a narrow resonance. A mass hierarchy of supersymmetric particles, where the $\tau$ sneutrino is the LSP, is assumed. In this model, the $\tau$ sneutrino can decay 
either into the final state under study, an $e \mu$ pair, via the couplings $\lambda_{132}$ and $\lambda_{231}$ or into $d \bar{d}$ via the coupling $\lambda_{311}^{\prime}$. For simplicity, we assume $\lambda_{132}=\lambda_{231}$ in all the presented results. Another theoretical interpretation is the Quantum blackhole $(\mathrm{QBH})$ signal that exhibits a broader signal shape with a sharp edge at the threshold mass $M_{\text {th }}$. Theories that allow for a fundamental Planck scale at the TeV scale offer the possibility of the production of microscopic black holes at the LHC. The production of a spin-0, colourless, charge-neutral QBH is considered, in a model with lepton flavour violation. In absence of any significant excess, the $95 \%$ CL limits on the signal cross section times branching ratio are calculated. For RPV $\tau$ sneutrino resonance signal, they are shown in Figure 6-left and the limits for the QBH signal are shown in Figure 6-right for $n=1(\mathrm{RS}), n=4,5,6(\mathrm{ADD})$ extra dimensions. A $\tau$ sneutrino LSP is excluded for masses below $1.0 \mathrm{TeV}$, for couplings $\lambda_{132}=\lambda_{231}=\lambda_{311}=0.01$, masses below $2.7 \mathrm{TeV}$ for couplings $\lambda_{132}=\lambda_{231}=\lambda_{311}=0.1$, masses below $3.3 \mathrm{TeV}$ for couplings $\lambda_{132}=\lambda_{231}=\lambda_{311}=0.2$. For QBH signals, the resulting expected and observed limits on threshold mass $\left(M_{t h}\right)$ for $n=1,4,5,6$ are $2.5 \mathrm{TeV}, 4.2 \mathrm{TeV}, 4.3 \mathrm{TeV}$ and $4.5 \mathrm{TeV}$ respectively.

\section{Heavy Majorana Neutrino}

Several extensions of the SM to address neutrino masses have been proposed so far, among which the seesaw mechanism is an appealing possibility. The seesaw mechanism introduces new heavy particles coupling to leptons and to the Higgs doublets, and accounts for the expected smallness of neutrino masses. Within the type-III seesaw model, the neutrino is considered a Majorana particle whose mass arises via the mediation of new massive fermions. These massive fermions are an SU(2) triplet of heavy Dirac charged leptons and a heavy Majorana neutral lepton. In proton-proton collisions, these massive fermions may be pair-produced through electroweak interactions in both charged-charged and charged-neutral pairs. A search for type-III seesaw signal in events with three or more electrons or muons is performed with $35.9 \mathrm{fb}^{-1}$ of data at $13 \mathrm{TeV}$ collected by the CMS experiment in 2016 [6]. Decays of heavy fermion pairs result in 27 different production processes and can naturally lead to
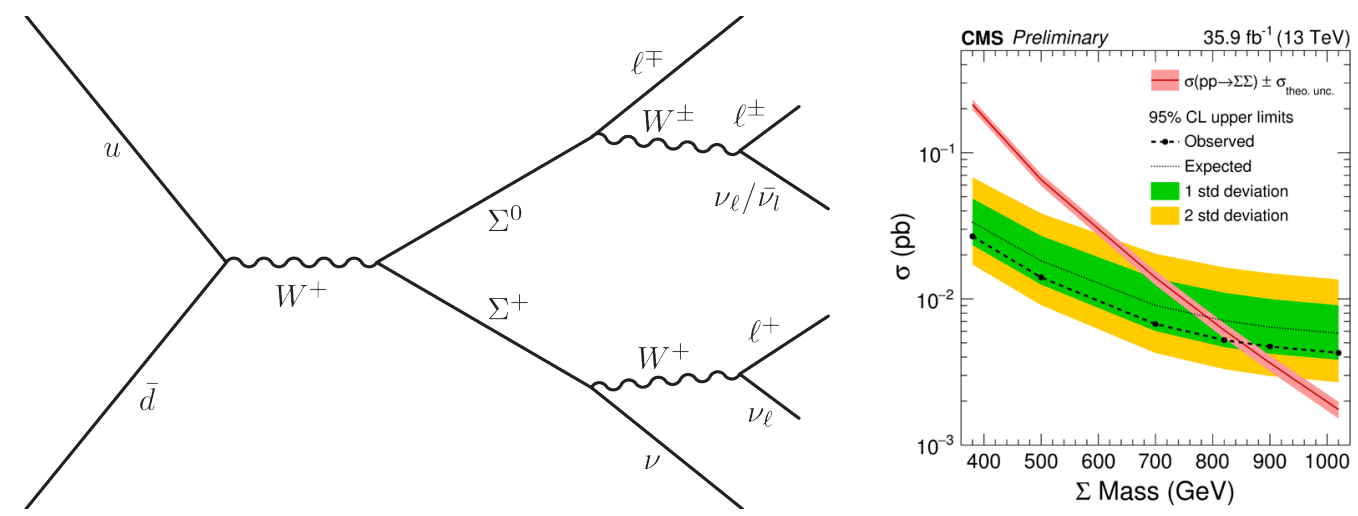

Figure 7. Left: Feynman diagram example of the heavy fermion production and decay in the type-III seesaw model [6]. Right: The 95\% confidence level upper limits on the cross section for production of heavy fermion pairs [6]. Also shown is the theoretical prediction for the cross section of the heavy fermion pair production via the type III seesaw mechanism, with its uncertainty. In the flavour-democratic scenario $\left(B_{e}=B_{\mu}=B_{\tau}\right)$, heavy fermion pair production is excluded for masses below $840 \mathrm{GeV}$.

multi-lepton final states, via decays of $\mathrm{W}, \mathrm{Z}$ or $\mathrm{H}$ bosons. An example Feynman diagram for one of 
the most relevant processes with three leptons in the final state is shown in Figure 7-left. As can be seen from the Feynman diagram, there is no LFV in the process itself, but the existence of a heavy fermion can enhance LFV processes like $\mu \rightarrow e \gamma$. The selected multi-lepton events are classified into statistically independent search channels using the number of leptons, lepton flavour and charge combinations, and other kinematic quantities. The number of opposite-sign-same-flavour(OSSF) pairs are counted, where the pairs are made using each lepton only once. Events are further classified in three categories as on-Z, below-Z, and above-Z, based on the presence of at least one OSSF pair with mass in the Z-mass window, below $81 \mathrm{GeV}$, or above $101 \mathrm{GeV}$, respectively. No statistically significant excess was found in the various signal regions that was probed. Assuming a flavourdemocratic scenario, 95\% confidence level upper limit on the cross section sum for the production of heavy fermion pairs is calculated, using the the CLs method. Based on the observed cross section limits, the production of heavy fermion pairs are ruled out for masses less than $850 \mathrm{GeV}$ (expected $790 \mathrm{GeV}$ ), as presented in Figure 7-right.

\section{Conclusion}

Some results on searches for charged lepton flavour violation, performed by the CMS experiment, using $8 \mathrm{TeV}$ and $13 \mathrm{TeV}$ pp collision data is presented. No significant excess has been observed in any of the searches and no hint of BSM has been found. This could be an indication that new physics beyond the Standard Model occurs at higher scales than the TeV scale. More precise answers can come only from indirect new physics probes, like precision studies of neutrinos and their properties, muon anomalous magnetic moment and searches for forbidden or suppressed processes like charged LVF. However, from the current searches, the limits are significantly extended with respect to previously published results. Further searches in more complicated final states are underway.

\section{References}

[1] S. Chatrchyan et al. [CMS Collaboration], "The CMS Experiment at the CERN LHC," JINST 3, S08004 (2008), doi:10.1088/1748-0221/3/08/S08004.

[2] CMS Collaboration, "Search for lepton flavour violating decays of the Higgs boson to $\mu \tau$ and e $\tau$ in proton-proton collisions at $\sqrt{s}=13 \mathrm{TeV}$," https://cds.cern.ch/record/2264540, CMS-PAS-HIG$17-001$.

[3] V. Khachatryan et al. [CMS Collaboration], "Search for lepton flavour violating decays of the Higgs boson to $e \tau$ and $e \mu$ in proton-proton collisions at $\sqrt{s}=8 \mathrm{TeV}$," Phys. Lett. B 763, 472 (2016) doi:10.1016/j.physletb.2016.09.062 [arXiv:1607.03561 [hep-ex]].

[4] S. Chatrchyan et al. [CMS Collaboration], "Search for top squarks in $R$-parity-violating supersymmetry using three or more leptons and b-tagged jets," Phys. Rev. Lett. 111, no. 22, 221801 (2013) doi:10.1103/PhysRevLett.111.221801 [arXiv:1306.6643 [hep-ex]].

[5] CMS Collaboration, "Search for high-mass resonances and quantum black holes in the $e \mu$ final state in proton-proton collisions at $\sqrt{s}=13 \mathrm{TeV}$," https://cds.cern.ch/record/2149046, CMS-PASEXO-16-001.

[6] CMS Collaboration, "Search for evidence of Type-III seesaw mechanism in multilepton final states in pp collisions at $\sqrt{s}=13 \mathrm{TeV}$," https://cds.cern.ch/record/2256657, CMS-PAS-EXO-17006. 